\title{
Optimal Transmit Antenna Selection using Hybrid Algorithm for Massive MIMO Technology
}

Charanjeet Singh ( $\square$ charanjeetsing17@gmail.com )

SRM University Haryana - Campus

P.C.Kishore Raja

SRM University Haryana - Campus

\section{Research Article}

Keywords: MIMO, Antenna Selection, Energy Efficiency, SLnO Algorithm, HS-WA Algorithm

Posted Date: July 31st, 2021

DOI: https://doi.org/10.21203/rs.3.rs-591271/v1

License: (c) (1) This work is licensed under a Creative Commons Attribution 4.0 International License. Read Full License 


\title{
Optimal Transmit Antenna Selection using Hybrid Algorithm for Massive MIMO Technology
}

\author{
${ }^{1}$ Charanjeet Singh, ${ }^{2}$ Dr. P.C.Kishore Raja \\ ${ }^{1}$ Research Scholar \\ Srm University, Delhi-Ncr, Sonepat, Haryana (India)-131029 \\ charanjeetsing17@gmail.com \\ ${ }^{2}$ Professor \\ Srm University, Delhi-Ncr, \\ Sonepat, Haryana (India)-131029.
}

\begin{abstract}
Massive Multiple Input Multiple Output (M-MIMO) systems specifically refers to a practical technique for sending and receiving more than one data signal simultaneously over the same radio channel by exploiting multipath propagation". It depends on several antennas for transferring varied data streams simultaneously. With the increase in count of antennas, the energy or power utilization also gets increased. Thus, it becomes necessary to select optimal transmit antennas that exist as the great challenge in M-MIMO systems. This work introduces a new "Hybrid Sea Lion-Whale Algorithm (HS-WA)" for selecting the optimal transmit antenna by considering the multi-objectives, which increases both capacity and efficiency. The adopted scheme is the combination of both "Whale Optimization Algorithm (WOA) and Sea Lion Optimization Algorithm (SLnO)" that optimizes the antenna's count and moreover, it finds out "which antenna to be selected". At last, the supremacy of presented model is confirmed over existing models in terms EE and capacity analysis.
\end{abstract}


Keywords: MIMO; Antenna Selection, Energy Efficiency; SLnO Algorithm; HS-WA Algorithm

\section{Nomenclature}

\begin{tabular}{|l|l|}
\hline Abbreviation & \\
\hline AS & Antenna Selection \\
\hline ASC & Average Secrecy Capacity \\
\hline BER & Bit-Error-Rate \\
\hline BS & Base Station \\
\hline BPSO & Binary Particle Swarm Optimization \\
\hline CNN & Convolutional Neural Network \\
\hline CDF & Cumulative Distribution Function \\
\hline CSI & Channel State Information \\
\hline DF & Decode-And-Forward \\
\hline EE & Energy Efficiency \\
\hline HS-WA & Hybrid Sea Lion-Whale Algorithm \\
\hline IVM & Import Vector Machine \\
\hline LUs & Legitimate Users \\
\hline MF & Match Filtering \\
\hline M-MIMO & Massive Multiple Input Multiple Output \\
\hline NB & Naive-Bayes \\
\hline OP & Outage Probability \\
\hline RF & Radio Frequency \\
\hline SNR & Signal To Noise Ratio \\
\hline SLnO & Sea Lion Optimization \\
\hline SVM & Support Vector Machine \\
\hline SE & Spectral Efficiency \\
\hline SM-MIMO-FDR & Spatial Modulation MIMO Full-Duplex Relaying \\
\hline SER & Symbol Error Rate \\
\hline SIC & Successive Interference Cancellation \\
\hline TAS & Transmit Antennas Selection \\
\hline WOA & Whale Optimization Algorithm \\
\hline ZF & Zero Forcing \\
\hline
\end{tabular}

\section{Introduction}

M-MIMO [9] [10], known as larger-scale MIMO depends on varied antennas for transferring various data streams in the network. Thus, it significantly raises the resolution and EE by exploiting several antennas at BS. By deploying numerous antennas at the receiver and/or transmitter, MIMO systems can [11] [12] accomplish enhanced spatial diversity. This 
improves the reliability during transmission and obtains high multiplexing gains that enhance system capacity and data rate. Accordingly, several RF chains are essential, which rapidly raises the hardware complexity and system cost. Therefore, to compute the cost and power consumption of RF chain, TAS is necessary [13] [14] [15] at both transmitting and receiving ends.

TAS in conventional systems are a foremost research problem for the past few decades. It reveals the improvements of the system with respect to EE, and moreover it depicts the process of deploying the antenna subset in communication. Along with SE, EE may also get improved that remains a challenging crisis for the upcoming networks [19] [20]. A promising method that reduces the aforesaid issue is optimal antenna selection. Optimal TAS adopts reasonable count of antennas at receiver or transmitter, and reduces the count of RF chains.

The measure of TAS could be the reduction of BER, maximization of SNR [21] or channel capacity at the receiver side and so on [8]. Choosing the optimal count of active RF chains and portraying the antenna formation improves the EE [22] [23]. In addition, more energy could be saved while RF chains of inactive antennas are turned off. Several TAS approaches like, Exhaustive search and norm-based TAS schemes were developed for improving EE in MIMO [4]. However, these approaches fail to attain a stable EE with respect to the count of transmission antennas [26]. The main contribution of the work is as follows:

- Introduces "Hybrid Sea Lion-Whale Algorithm", which is the hybridized edition of WOA as well as SLnO models.

The paper is arranged as follows: Section II shows the review on existing models. Section III explains the system model for optimal TAS. Section IV depicts the hybrid sea lion-whale algorithm for TAS in M-MIMO. Section V analyses outcomes and section VI concludes the work. 


\section{Literature Survey}

\subsection{Related Works}

In 2020, Cao et al. [1] presented an effectual approach for TAS in "SM-MIMO-FDR systems", in which the relay exploits DF protocol. In addition, the accurate SER as well as OP were derived for indicating the performance of adopted system. The experimentations have revealed that the proposed method significantly enhanced the efficiency over the compared schemes.

In 2020, Khalid et al. [2] have designed a joint TAS mechanism along with pre-coding mechanism. Accordingly, heuristic approaches with minimal complexity were deployed to carry out the antenna selection for limiting the count of active antennas. Therefore, lessening the power utilization consequently enlarges the EE. Moreover, SIC oriented technique was exploited for pre-coding the chosen transmit antennas. Furthermore, the outcomes demonstrated that the designed model was really effective with minimal complexity and high EE.

In 2019, Moualeu et al. [3] have presented an enhanced framework for analysing the MIMO systems at $\alpha-\mu$ fading conditions. Subsequently, 2 scenarios were considered here; the transmitter has awareness regarding the CSI and (b) the transmitter has no awareness regarding CSI of wiretap connection. In addition, two new techniques were suggested for deriving a systematic analysis of the ASC. Further, the superiority of proposed technique was proved in terms of high SNR.

In 2018, Eskandari et al. [4] developed a new solution for solving power allocation issues and for maximizing EE in point-to-point MIMO systems. Subsequently, an optimum solution was derived that revealed the association among the optimal EE and system constraints like channel states and circuit power conditions. Moreover, an upper bound was derived and 
based on that bound, a novel TAS model was proposed to attain optimal solution. Further, the performance of the developed technique was analyzed in terms of complexity.

In 2018, Asaad et al. [5] have analysed the impact of TAS on confidentiality performance of M-MIMO schemes. In addition, a wiretap setting was considered, where a predetermined count of antennas were chosen and the secret data were passed to a legitimate receiver. Moreover, a precise estimation of the instantaneous secrecy rate was derived, by which the secrecy could be enhanced. Finally, the simulated outcomes have demonstrated the enhancement of presented technique with high accuracy.

In 2020, Tran et al. [6] have established 2 TAS solutions, "Solutions I and II" for modelling a secure TAS communication model. Particularly, these solutions focused on increasing the receiver signal power among nearer user and source and among distant user and source. Consequently, asymptotic and accurate expression was derived for the confidential OP of overall system and LUs. At last, the attained outcomes illustrated the superiority of solution II over solution I in terms of overall secrecy.

In 2018, He et al. [7] have focused on the scenario, in which the source adopted TAS as the transmission approach. Here, it was assumed that the CSI of authenticated receiver was known to source, whereas, the CSI of eavesdrop could be unknown or known to source. Moreover, by considering TAS as a classification issue, two approaches namely, NB and SVM have been proposed. Experimentation was done with the proposed work, and its performance was compared over the traditional model in terms of overhead.

In 2020, Yang and Zhao [8] have presented a novel multi-class IVM oriented framework for maximizing the average received SNR. In fact, TAS was known to be a capable approach for reducing the cost and it also increases the gain of the MIMO system. Furthermore, the analysis has demonstrated the superiority of introduced model, which has acquired minimal complexity with high SNR over other compared models. 
TABLE I. Review on existing M-MIMO Antenna Selection techniques

\begin{tabular}{|c|c|c|c|}
\hline Author & Methods & Features & Challenges \\
\hline $\mid \begin{array}{l}\text { Nguyen et al. } \\
{[1]}\end{array}$ & $\begin{array}{l}\text { SM-MIMO- } \\
\text { FDR }\end{array}$ & $\begin{array}{l}\text { Better throughput } \\
\text { Reduced error rate }\end{array}$ & $\begin{array}{l}\text { Hardware impairment may } \\
\text { occur. } \\
\text { Self interference is not } \\
\text { eliminated completely. }\end{array}$ \\
\hline Khalid et al. [2] & BPSO & $\begin{array}{l}\text { Better SE } \\
\text { Offers high spatial gain }\end{array}$ & $\begin{array}{l}\text { No consideration on RF } \\
\text { chain selection. }\end{array}$ \\
\hline $\begin{array}{l}\text { Moualeu et al. } \\
{[3]}\end{array}$ & CDF & $\begin{array}{l}\text { High SNR } \\
\text { Reduced fading }\end{array}$ & $\begin{array}{l}\text { No consideration on } \\
\text { varied fading scenarios. } \\
\text { Need more focus on } \\
\text { capacity loss }\end{array}$ \\
\hline $\begin{array}{l}\text { Eskandari et al. } \\
\text { [4] }\end{array}$ & $\begin{array}{l}\text { Water-filling } \\
\text { algorithm }\end{array}$ & $\begin{array}{l}\text { High EE } \\
\text { Reduced complexity }\end{array}$ & $\begin{array}{lll}\text { EE decreases with } \\
\text { increase in distance. }\end{array}$ \\
\hline Asaad et al. [5] & $\begin{array}{l}\text { Gaussian based } \\
\text { model }\end{array}$ & $\begin{array}{l}\text { Minimal complexity } \\
\text { Improved } \quad \text { secrecy } \\
\text { performance }\end{array}$ & $\begin{array}{l}\text { Noise generation should } \\
\text { be combined with TAS for } \\
\text { more confidentiality. }\end{array}$ \\
\hline Tran et al. [6] & CDF function & $\begin{array}{l}\text { Reduced interference } \\
\text { Enhanced secrecy } \\
\text { performance }\end{array}$ & $\begin{array}{l}\text { Difficult to accomplish on } \\
\text { realistic scenarios. }\end{array}$ \\
\hline He et al. [7] & SVM classifier & 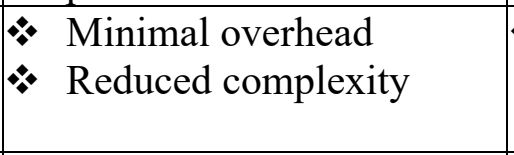 & $\begin{array}{l}\text { Performance } \\
\text { degraded if only partial } \\
\text { CSI is available. }\end{array}$ \\
\hline $\begin{array}{l}\text { Yang and Zhao } \\
{[8]}\end{array}$ & IVM & $\begin{array}{l}* \text { Minimal complexity } \\
\text { * High SNR }\end{array}$ & $\begin{array}{l}\text { No consideration on error } \\
\text { factors. }\end{array}$ \\
\hline
\end{tabular}

\subsection{Review}

Table 1 demonstrate the review on TAS in MIMO systems. Initially, SM-MIMO-FDR model was presented in [1] that offers accurate throughput and it reduced the error rate. Nevertheless, hardware impairment may occur. BPSO was developed in [2] that offers high spatial gain with better SE, but it have to consider more on RF chain selection. In addition, CDF was used in [3], which provides minimal fading and it presents high SNR; nevertheless, it should focus on capacity loss. Also, Water-filling algorithm was employed in [4] that provide high EE with reduced complexity. However, EE decreases with increase in distance. Likewise, Gaussian based model was presented in [5] that offer enhanced secrecy performance with minimal complexity. However, noise generation should be combined with 
TAS for more confidentiality. In addition, CDF function was deployed in [6] that ensure reduced interference and it offers enhanced secrecy performance. Nevertheless, it is difficult to accomplish on realistic scenarios. SVM classifier was deployed in [7] that provide minimal overhead and reduced complexity, however, performance gets degraded if only partial CSI is available. IVM was presented in [8] that offer reduced complexity with high SNR; but, there is no consideration on error factors.

\section{Optimal TAS for MIMO Technology}

\subsection{System Model}

Assume an isolated cell with one BS and $A$ terminals. The TX antennas in $O_{t}$ counts are integrated in BS and all $A$ includes separate $\mathrm{RX}$ antennas. The received signal vectors at $A$ is indicated in Eq. (1), where $E$ refers to $A \times O_{t}$ small scale Rayleigh fading channel matrix, $s$ refers to $A \times 1$ received vector for all $A, P_{t x}$ refers to all TX power at forward link, $\varsigma$ refers to TX power normalization factor, which is evaluated as in $\varsigma \approx \sqrt{\frac{O_{t}}{A}} \cdot s v$ refers to $A \times 1$ signal vector, $n v$ refers to $A \times 1 \mathrm{AWGN}$ noise vector and $V$ refers to $O_{t} \times A$ pre-coding matrix to reduce IUI. Accordingly, 2 pre-coding matrices are formulated for LS-MIMO, namely, MF $\left(M F: V=O_{t}^{-1} B^{B}\right)$, and $\mathrm{ZF}\left(Z F: V=B^{B}\left(B B^{B}\right)^{-1}\right)$.

$$
s_{m}=\sqrt{P_{t x}} B \varsigma V s v+n v
$$

Suppose, if transmitter has idyllist CSI, then the system is termed as LS-MIMO. For computing the EE of LS-MIMO BS, there should be a proper design for power consumption. Eq. (2) defines the sum power, $P_{\text {sum }}$ as a tractable power utilization technique, here $P_{P A}$ and $P_{B A}$ refers to power utilization of Power Amplifier (PA) and baseband (BB), and the RF front-end power consumption is pointed out as $P_{R F_{\text {fromt }}}$. 


$$
P_{\text {sum }}=P_{P A}+P_{B A}+E_{t} P_{R F_{\text {front }}}
$$

In addition, power utilization $P_{c p}$ is evaluated, which increase with increase in $O_{t}$ as shown in Eq. (3). Consequently, Eq. (3) is redefined as in Eq. (4).

$$
\begin{aligned}
& P_{c p}=\left(P_{B A} / O_{t}+P_{R F_{\text {front }}}\right) \\
& P_{\text {sum }}=P_{P A}+O_{t} P_{c p}
\end{aligned}
$$

Accordingly, consider OFDM system (10MHz) having "Class-B efficiency (78:5\%) with subcarriers (1024), and IBO as $11 \mathrm{~dB}$ ”. Based on these parameters, the PA efficiency is assumed as $22 \%$. The association among $P_{P A}$ and $P_{t x}$ is given in Eq. (5).

$$
P_{t x}=\eta P_{P A}
$$

As $P_{B A}$, the LSMIMO BB evaluation design, $s v(U$ flops $) P_{B A}$ is addressed in [11].

$$
z=O_{t} S .\left[\begin{array}{l}
\left(\frac{C_{g i}}{C_{s d}}\right) \log _{2}\left(C_{g i} S\right)+\left(\frac{C_{g i}}{C_{s d}}\right)\left(1-\frac{C_{p t}}{C_{s t}}\right) A+\left(\frac{C_{g i}}{C_{s d}}\right) \\
\left(\frac{C_{p t}}{C_{s t}}\right) \log _{2}\left(\frac{C_{g i} C_{p t}}{C_{s d} C_{d l}}\right)+\left(\frac{C_{d l}}{C_{s t}}\right) A^{2}
\end{array}\right]
$$

All constraints in Eq. (6) are explained below.

- Parameter $S$ refers to bandwidth with $10 \mathrm{MHz}$ power utilization.

- Parameter $C_{s t}$ refers to slot length with $0.5 \mathrm{~ms}$ power utilization.

- Parameter $C_{p t}$ refers to pilot length in a single slot with $0.214 \mathrm{~ms}$ power utilization.

- Parameter $C_{s d}$ refers to symbol duration with $0.214 \mathrm{~ms}$ power utilization.

- Parameter $C_{c o}$ refers to GI with $4.7 \mu$ s power utilization.

- Parameter $C_{g i}$ refers to GI having $66.7 \mu$ s power utilization.

- Parameter $C_{d l}$ refers to delay spread of GI with $4.7 \mu$ s power utilization.

The correlation among $P_{B A}$ and $s v(U$ flops $)$ is shown in Eq. (7), where VLSI efficiency is referred as $\varpi$, and $\varpi=5 U$ flop $/ R$ and $50 U$ flop $/ R$ was chosen. 


$$
P_{B A}=\frac{s v(U \text { flops })}{\varpi(U \text { flops } / R)}
$$

\subsection{Optimal Selection of Transmit Antenna}

The received signal by $m^{\text {th }}$ user is shown in Eq. (8), wherein, the $1 \times E_{t}$ channel vector of $m^{\text {th }}$ user is signified as $g_{k}$, and pre-coding vector for $m^{\text {th }}$ user is signified as $p:,_{k}$. The last term in Eq. (8) is called IUI.

$$
q_{k}=\sqrt{\frac{P_{t x} E_{t}}{B}} g_{k},: p:,_{k} z_{k}+e_{k}+\sqrt{\frac{P_{t x} E_{t}}{B}} \sum_{l \neq k} g_{k},: p:,_{l} z_{l}
$$

The capability of one isolated cell is revealed in Eq. (9), here $\alpha$ refers to scaling factor, $O_{0} S$ refers to noise power at bandwidth $S$.

$$
M=\alpha S . \sum_{k=1}^{K} D\left[\log _{2}\left(1+\frac{\frac{P_{t x} O_{t}}{A}\left|g_{k},: p:, k\right|^{2}}{\left.\frac{P_{t x} O_{t}}{A}\left|\sum_{l \neq k} g_{k},: p:, l\right|\right|^{2}+O_{0} S}\right)\right]
$$

Eq. (9) is formulated when the system moves to LS MIMO area, (i.e. $O_{t}>10 A$ ). In Eq. (10), $X$ refers to IUI. EE is modelled as per Eq. (11). Further, the optimal TX antenna count $O_{t}^{\text {opt }}$ must satisfy Eq. (12).

$$
\begin{aligned}
& S_{\text {approx }}^{L S-M I M O} \approx \alpha S A .\left[\log _{2}\left(1+\frac{P_{t x} O_{t}}{\left(X+O_{0} S\right) A}\right)\right] \\
& E E=M / P_{\text {sum }}
\end{aligned}
$$




$$
\begin{aligned}
& \frac{\partial}{\partial E_{t}} E E=\frac{\partial}{\partial E_{t}}\left(\frac{M_{\text {approx }}}{\frac{1}{\eta} P_{t x}+O_{t} P_{c p}}\right) \\
& =\frac{S A P_{t x}}{\left(X+O_{0} S\right) A\left(1+\frac{P_{t x} O_{t}}{\left(L+O_{0} S\right) A}\right) P_{\text {sum }} \log _{2} p}- \\
& \frac{S A P_{c p} \cdot \log _{e}\left(1+\frac{P_{t x} O_{t}}{\left(X+O_{0} S\right) A}\right)}{P_{\text {sum }}^{2} \log _{2} p} \\
& =0
\end{aligned}
$$

Thereby, $O_{t}^{o p t}$ is computed as in Eq. (13), where $\Gamma=\frac{P_{t x}^{2}-A O_{0} S P_{c p}-A X P_{c p}}{A\left(X+O_{0} S\right) P_{c p} \exp (1)}$ and $R$ represents the lambert function and is expressed as $\Gamma=R(\Gamma) \exp (R(\Gamma))$.

$$
O_{t}^{o p t} \approx \frac{\left(X+O_{0} S\right) A}{P_{t x}}(-1+\exp (1+R(\Gamma)))
$$

Using Eq. (13), $O_{t}^{\text {opt }}$ could be attained, however, the challenge is "how to select the $O_{t}^{\text {opt }}$ antennas from total number of antenna $O_{t}^{\text {total }}$. The channel capacity of $O_{t}^{\text {opt }}$ when choosing columns of $B$ are $\left\{b_{1}, b_{2}, \ldots b_{O_{t}^{\text {opt }}}\right\}, M_{\left\{b_{1}, b_{2}, \ldots b_{o_{t}^{\text {opt }}}\right\}}$ is given in Eq. (14), where, $1 \times O_{t}^{\text {opt }}$ channel vector for $k^{\text {th }}$ user is referred as $g_{k,\left\{b_{1}, b_{2}, \ldots b_{E_{t}^{o p t}}\right\}}, O_{t}^{\text {opt }} \times 1$ pre-coding vector referred as $p_{\left\{b_{1}, b_{2}, \ldots . b b_{t}^{\text {opt }}\right\}, k}$ when $\left\{b_{1}, b_{2}, \ldots b_{O_{t}^{\text {opt }}}\right\}^{\text {th }}$ is chosen.

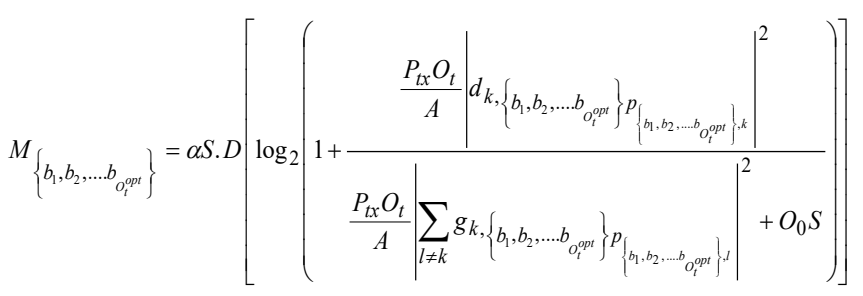

On considering green BS, EE should be maximized instead of increasing the capacity. In Eq. (11), the optimal AS for EE and channel capacity are same, as $O_{t}^{\text {opt }}$ was already chosen from Eq. (13). Thus, $P_{\text {sum }}$ turns out to be independent of AS. For optimally selecting the 
antennas, $O_{t}^{\text {opt }}$ columns with higher $\mathrm{EE}$ is chosen as per Eq. (15), where, all feasible combinations of $O_{t}^{o p t}$ antennas is referred as $L$.

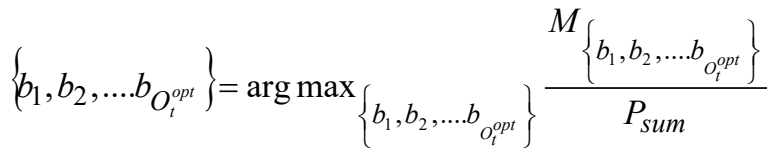

\section{Hybrid Sea Lion-Whale Algorithm for TAS in M-MIMO}

\subsection{Solution Encoding}

The proposed work concerns on electing the optimal count of antennas and it finds out "which antenna should be selected". This optimization problem is solved by a new hybrid algorithm and the input solution to the algorithm is illustrated in Fig 1. The antennas $b$ are provided as solutions and chromosome length depends on antenna count $E_{t}$. In Fig 1, "0" indicates unselected antennas and " 1 " depicts the selected antennas. Thus, the optimal count and the appropriate antenna to be selected is determined.

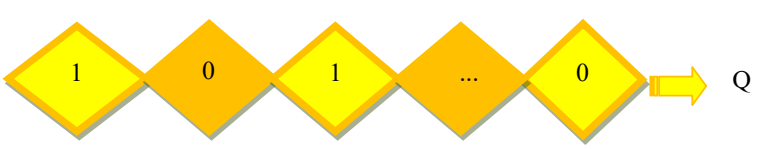

Fig. 1. Solution Encoding

\subsection{Proposed WTU Algorithm}

For enhancing the traditional WOA [25] [29] [30], it is intended to hybridize the concept of SLnO [24] with WOA. In fact, the hybrid optimizations are reported to be capable for solving search issues [26] [31]. The arithmetical model of adopted HS-WA algorithm is described here.

(i) Encircling Prey: As per the presented HS-WA model, the update occurs depending on random number $\phi$. If $\phi$ is lesser than $0.5(\phi<0.5)$, and if $(|I|<1)$ and if random integer, $r a$ is less than 0.5 (i.e. $r a<0.5$ ), the update takes place as per encircling phase of WOA, i.e. as 
shown in Eq. (17). In Eq. (16) and Eq. (17), $\vec{I}$ and $\vec{H}$ refer to the coefficient vectors and present iteration is signified by $t$.

$$
\begin{aligned}
\vec{G} & =\left|\vec{H} \cdot \vec{Q}_{p}(t)-\vec{Q}(t)\right| \\
\vec{Q}(t+1) & =\vec{Q}_{p}(t)-\vec{I} \cdot \vec{G}
\end{aligned}
$$

Also, $\vec{Q}$ denote position vector and $\vec{Q}_{p}$ signifies best position. Furthermore, $\vec{I}$ and $\vec{H}$ are evaluated by Eq. (18) and Eq. (19). In Eq. (18), the component $\vec{a}$ is diminished from 2 to 0 for further iterations. The arbitrary vectors $r a_{1}$ and $r a_{2}$ lies among [0, 1].

$$
\begin{aligned}
& \vec{I}=2 \widehat{a} \cdot r a_{1}-\vec{a} \\
& \vec{H}=2 r a_{2}
\end{aligned}
$$

On the other hand, if $r a>0.5$, the update occurs as per detecting phase of SLnO as given in Eq. (20), in which $D$ denotes the distance among sea lion and target prey, $\vec{H}$ refers to random integer, $\vec{M}(t)$ refers to the position of target prey.

$$
\vec{Q}(t+1)=\vec{M}(t)-D \cdot \vec{H}
$$

If $(|I|>1)$, and if $r a<0.5$, the update occurs as per exploration phase of WOA, i.e. as shown in Eq. (22), where the arbitrary position vector elected from the present population is indicated by $X_{(\text {rand })}$.

$$
\begin{array}{r}
\vec{G}=\left|\vec{H} \vec{Q}_{(\text {rand })}-\vec{Q}\right| \\
\vec{Q}(t+1)=\vec{Q}_{(\text {rand })}-\vec{I} \cdot \vec{G}
\end{array}
$$

On the other hand, if $r a>0.5$, the update takes place based on exploration phase of SLnO as shown in Eq. (23), where $\overrightarrow{Q_{r n d}}(t)$ refers to the arbitrary sea lion chosen from present population.

$$
\vec{Q}(t+1)=\overrightarrow{Q_{r n d}}(t)-D \vec{H}
$$


Likewise, if $\phi>0.5$, and if $r a<0.5$, the update occurs as per exploitation phase of WOA, i.e. as given in Eq. (24). Here, $\vec{G}$ refers to distance of $i^{t h}$ whale to prey and is given in Eq. (25), $b$ denotes "logarithmic spiral shape" and $l$ is an arbitrary variable between $[-1,1]$.

$$
\begin{aligned}
& Q(t+1)=\vec{G}^{\prime} e^{b l} \cdot \operatorname{Cos}(2 \pi l)+\vec{Q}_{p}(t) \\
& G^{\prime}=\left|\vec{Q}_{p}(t)-\vec{Q}(t)\right|
\end{aligned}
$$

Conversely, if $r a>0.5$, the update occurs as per exploitation phase of SLnO as given in Eq. (26), here the distance amongst target prey and sea lion is denoted by $\vec{M}(t)-\vec{Q}(t)$, the absolute value is denoted by $\mid$ and the random number among to 1 is referred by $l$.

$$
\vec{Q}(t+1)=|\vec{M}(t)-\vec{Q}(t) \cdot \cos (2 \pi l)|+\vec{M}(t)
$$

\begin{tabular}{|l|}
\hline \multicolumn{1}{|c|}{ Algorithm 1: Proposed HS-WA model } \\
\hline Assign the population of whales $Q_{i}(i=1,2, \ldots ., n)$ \\
Measure the fitness of all exploring agents \\
$Q^{*}$ is the best agent for exploring \\
While $t$ is less than the number of iterations \\
For every search agents \\
Update $a, I, H, \phi$ and $l$ \\
if $1 \phi<0.5$ \\
if $(|I|<1)$ \\
if $r a<0.5$ \\
$\mid$ Update position as in Eq. (17) \\
else \\
$\mid$ Update position as in Eq. (20), i.e. based on detecting phase of SLnO \\
end if \\
else if \\
If $r a<0.5$ \\
Update position as in Eq. (22) \\
else \\
$\mid$ Update position as in Eq. (23), i.e. based on exploration phase of SLnO \\
end if \\
end if \\
else \\
if $r a<0.5$ \\
Update position as in Eq. (24) \\
else \\
$\mid$ Update position as in Eq. (26), i.e. based on exploitation phase of SLnO \\
end if \\
end for
\end{tabular}


Measure the fitness of exploring agent as per Eq. (15)

if better solution is attained, update $Q^{*}$

$t=t+1$

end while

return $Q^{*}$

\section{Results and Discussions}

\subsection{Simulation Setup}

The proposed optimal TAS model was implemented in "MATLAB" and the outcomes were noticed. Accordingly, the supremacy of the presented HS-WA model was evaluated over existing models namely, MV-GSA [27], I-SLnO [28], SLnO [24] and WOA [25]. Here, analysis was done using 2 setups. The first setup was " $P_{t x}=40 \mathrm{~W}$, $s v(U$ flop $/ R)=5 U$ flop $/ R, P_{R F_{\text {front }}}=975 \mathrm{~mW}$ and the $\quad 2^{\text {nd }} \quad$ setup $P_{t x}=10 \mathrm{~W}, P_{\text {sum } 1} \operatorname{sv}(U$ flop $/ R)=50 U$ flop $/ R, P_{R F_{\text {front }}}=97.5 \mathrm{~mW}$ ". Moreover, the analysis was carried out for MF coding and ZF coding with respect to relative efficiency and capacity.

\subsection{Analysis on Capacity}

The capacity analysis of adopted HS-WA scheme over existing schemes like MV-GSA, ISLnO, SLnO and WOA is given in Fig. 2. The analysis is done for MF and ZF under two set ups by varying the count of users from 1 to 8 . From the analysis, the presented model has revealed higher capacity value over other compared models. Initially, the capacity is lower for lesser count of users; however, as the count of users increases, the capacity has reached a higher level. Particularly, for ZF under set up 2 (from Fig. 2(d)), the HS-WA scheme has obtained a higher capacity, whereas, for other experimentations, the proposed work has exhibited a comparatively lower capacity values. More specifically, the implemented model for MF under set up 1 is $48.89 \%, 15.56 \%, 15.56 \%$ and $2.22 \%$ improved than traditional MVGSA, I-SLnO, SLnO and WOA when user count is 7. Likewise, the capacity analysis of the 
developed HS-WA model over conventional schemes for ZF under $2^{\text {nd }}$ set up is illustrated in Fig. 2 (d), where the proposed HS-WA scheme has accomplished a higher capacity of 7.3. That is, the performance of adopted model is $65.75 \%, 1.37 \%, 17.81 \%$ and $1.37 \%$ improved than traditional MV-GSA, I-SLnO, SLnO and WOA when user count is 8. Altogether, from the analysis, the presented scheme has found to be effective with higher capacity.

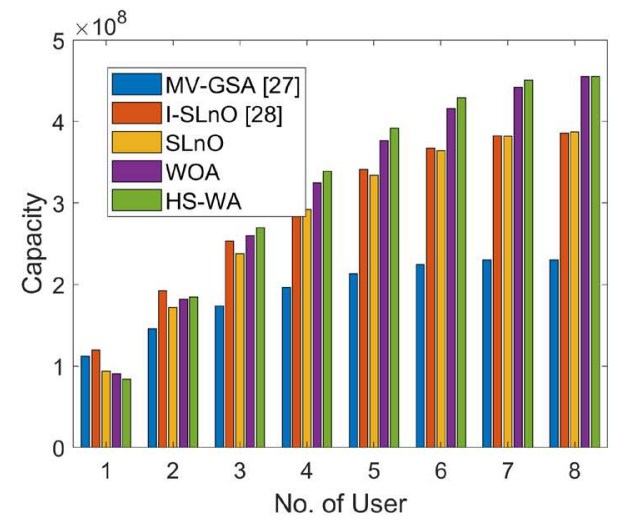

(a)

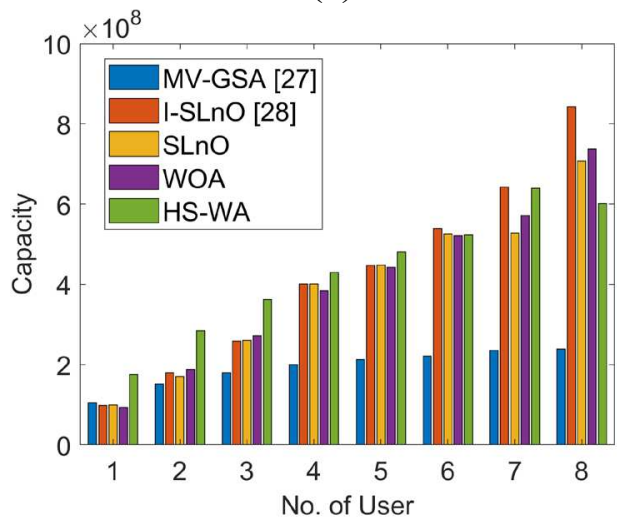

(c)

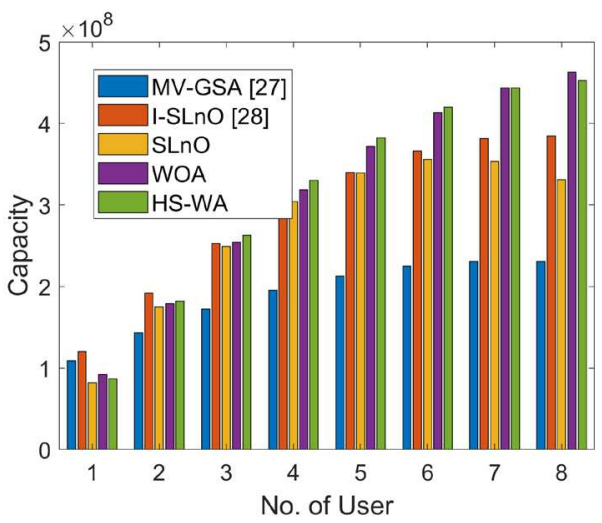

(b)

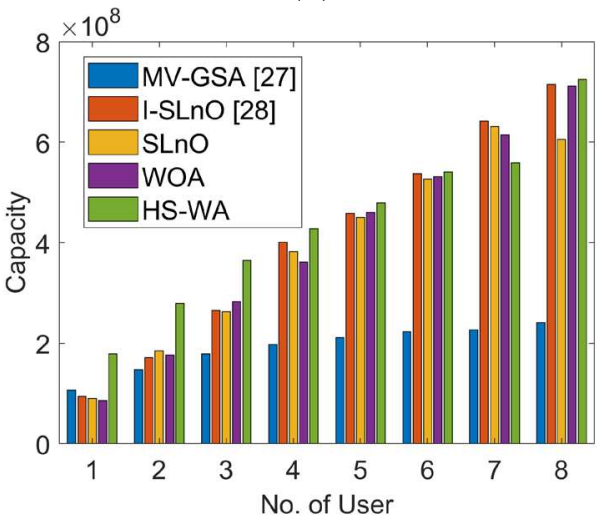

(d)

Fig. 2. Capacity analysis of adopted model over existing models for (a) MF (b) ZF for setup

$$
1 \text { and (c) MF (d) ZF for setup } 2
$$

\subsection{Energy efficiency Analysis}

The analysis on relative efficiency attained by the developed HS-WA model over existing models is analysed in this section. The analysis is carried out for ZF and MF for varied number of users' ranges from 5, 10, 15, 20, 25, 30, 35 and 40. On observing Fig. 3, the adopted work has attained a higher efficiency than the compared models for both the set ups. 
In particular, the adopted HS-WA model has achieved a higher efficiency of 0.979 ; while, the compared approaches has revealed a comparatively lower efficiency. From Fig. 3(c), the adopted model for MF under set up 2 is $0.92 \%, 0.102 \%, 0.102 \%$ and $0.102 \%$ superior to other existing models like MV-GSA, I-SLnO, SLnO and WOA, when the number of users is 40. Moreover, from Fig. 3(d), the implemented scheme for ZF under set up 2 has achieved a higher efficiency value of 0.979 , whereas the compared models have achieved a relatively lower efficiency of 0.97 . That is, the developed model is $0.92 \%, 0.102 \%, 0.102 \%$ and $0.102 \%$ superior to other existing models like MV-GSA, I-SLnO, SLnO and WOA, when the number of users is 40 . Therefore, the improved efficiency accomplished by the developed HS-WA model has been proved effectively.

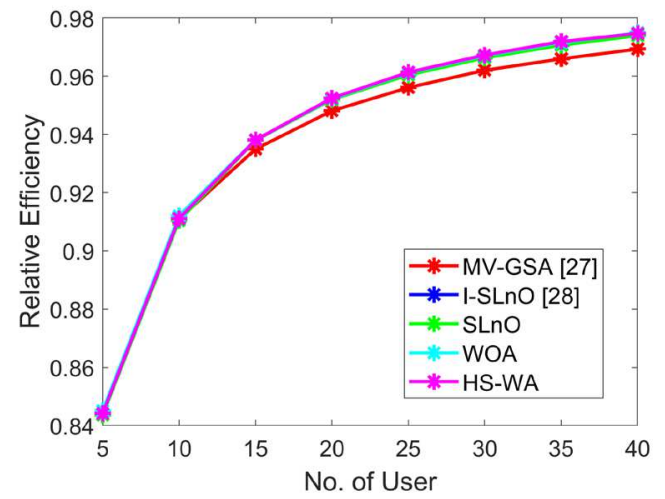

(a)

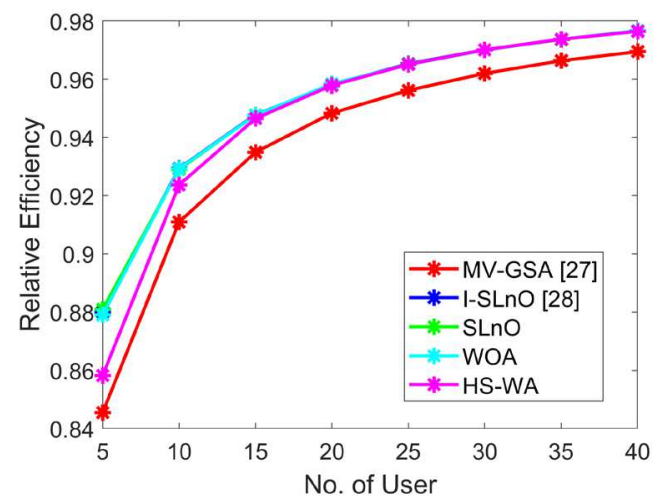

(c)

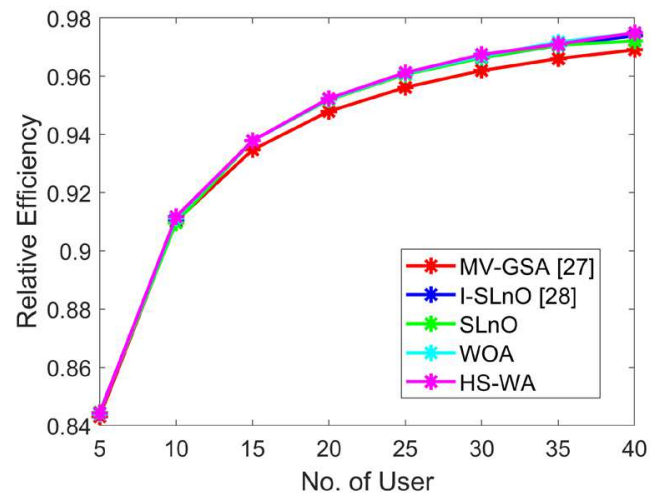

(b)

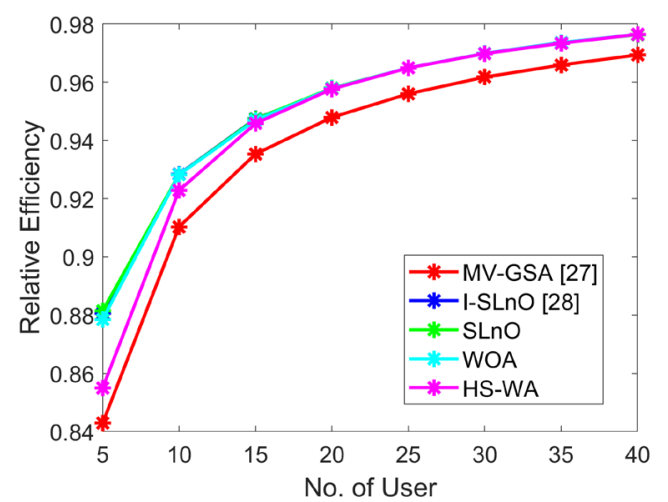

(d)

Fig. 3. Analysis on relative efficiency by adopted model over existing models for (a) MF (b) ZF for setup 1 and (c) MF (d) ZF for setup 2 


\subsection{Analysis on Optimal Transmit Antenna Count}

Table II and Table III reveals the optimal count of transmitted antennas attained by the presented approach and conventional approaches for varied number of users that ranges from $5,10,15,20,25,30,35$ and 40 . Table II portrays the optimal count of antennas obtained by developed HS-WA and existing models for ZF and MF under set up 1. In addition, Table III exhibits the optimal count of antennas attained by the presented and existing models for $\mathrm{ZF}$ and MF under set up 2. From the results, it was observed that the optimal antenna count varies for $\mathrm{ZF}$ and MF depending on the count of users.

TABLE II. Optimal count of transmit antenna by implemented model over existing models for setup 1

\begin{tabular}{|r|r|r|r|r|r|}
\hline \multicolumn{7}{|c|}{ Match Filtering } \\
\hline No of User & MV-GSA [27] & I-SLnO [28] & SLnO & WOA & HS-WA \\
\hline 5 & 4448 & 5066 & 7579 & 3459 & 3796 \\
\hline 10 & 4453 & 7500 & 5038 & 5335 & 5115 \\
\hline 15 & 4331 & 7516 & 7435 & 6612 & 6534 \\
\hline 20 & 4382 & 7563 & 7580 & 7433 & 7752 \\
\hline 25 & 4357 & 7509 & 7509 & 7385 & 7832 \\
\hline 30 & 4352 & 7649 & 7530 & 7364 & 7597 \\
\hline 35 & 4293 & 7522 & 7492 & 7527 & 7988 \\
\hline 40 & 4296 & 7553 & 7553 & 7550 & 7337 \\
\hline \multicolumn{5}{|c|}{ Zero Forcing } \\
\hline No of User & MV-GSA [27] & I-SLnO [28] & SLnO & WOA & HS-WA \\
\hline 5 & 4431 & 7514 & 5005 & 3335 & 3464 \\
\hline 10 & 4281 & 7600 & 4125 & 5426 & 5253 \\
\hline 15 & 4321 & 7445 & 7490 & 6679 & 6474 \\
\hline 20 & 4319 & 7487 & 7487 & 7173 & 7548 \\
\hline 25 & 4371 & 7589 & 7589 & 7194 & 7632 \\
\hline 30 & 4376 & 7513 & 7555 & 7215 & 7865 \\
\hline 35 & 4342 & 7544 & 7580 & 7739 & 6934 \\
\hline 40 & 4290 & 7533 & 8576 & 7602 & 7737 \\
\hline
\end{tabular}

TABLE III. Optimal count of transmit antenna by implemented model over existing models for setup 2 


\begin{tabular}{|r|r|r|r|r|r|}
\hline No of User & MV-GSA [27] & I-SLnO [28] & SLnO & WOA & HS-WA \\
\hline 5 & 9156 & 4919 & 4919 & 9121 & 9142 \\
\hline 10 & 9088 & 4981 & 8084 & 9071 & 9076 \\
\hline 15 & 9065 & 4906 & 4976 & 9052 & 9053 \\
\hline 20 & 9052 & 7523 & 4982 & 9042 & 9042 \\
\hline 25 & 9044 & 9035 & 7507 & 9035 & 9035 \\
\hline 30 & 9038 & 9030 & 7707 & 9030 & 9030 \\
\hline 35 & 9034 & 9026 & 4987 & 9026 & 9026 \\
\hline 40 & 9030 & 8726 & 7462 & 9023 & 9024 \\
\hline \multicolumn{7}{|c|}{ Zero Forcing } \\
\hline No of User & MV-GSA [27] & I-SLnO [28] & SLnO & WOA & HS-WA \\
\hline 5 & 9159 & 8256 & 9750 & 9121 & 9145 \\
\hline 10 & 9090 & 4184 & 9072 & 9072 & 9077 \\
\hline 15 & 9065 & 7403 & 8838 & 9053 & 9054 \\
\hline 20 & 9052 & 7487 & 9042 & 9042 & 9042 \\
\hline 25 & 9044 & 9035 & 4991 & 9035 & 9035 \\
\hline 30 & 9038 & 6436 & 9030 & 9030 & 9030 \\
\hline 35 & 9034 & 8357 & 9026 & 9026 & 9027 \\
\hline 40 & 9030 & 6968 & 9024 & 9024 & 9024 \\
\hline
\end{tabular}

\section{Conclusion}

This paper has developed a novel algorithm for choosing the optimal transmit antenna, by which the capacity and relative efficiency was increased. In order to carry out optimal TAS, a novel algorithm namely HS-WA was introduced, which optimally selected the antenna count and it also determined "which antenna to be chosen". At last, the superiority of presented scheme was proved over the existing models in terms of efficiency and capacity. On observing the analysis outcomes, the adopted model for MF under set up 2 was $0.92 \%$, $0.102 \%, 0.102 \%$ and $0.102 \%$ superior to other existing models like MV-GSA, I-SLnO, SLnO and WOA, when the number of users was 40. Moreover, the implemented scheme for ZF under set up 2 has achieved a higher efficiency value of 0.979 , whereas the compared models have achieved a relatively lower efficiency of 0.97 . i.e. the developed model was $0.92 \%$, $0.102 \%, 0.102 \%$ and $0.102 \%$ superior to other existing models like MV-GSA, I-SLnO, SLnO and WOA, when the number of users was 40 . Thereby, the enhancement of the developed model has been proved over the conventional works. 


\section{References}

[1] Ba Cao Nguyen, Tran Manh Hoang, Phuong T. Tran, Vinh P. Nguyen, "Transmit antenna selection for spatial modulation MIMO IoT networks with full-duplex relaying”, AEU - International Journal of Electronics and Communications, vol. 123, Article 153312, August 2020.

[2] Salman Khalid, Rashid Mehmood, Waqas Bin Abbas, Farhan Khalid, Muhammad Naeem, "Joint transmit antenna selection and precoding for millimeter wave massive MIMO systems”, Physical Communication, vol. 42, Article 101137, October 2020.

[3] J. M. Moualeu, D. B. da Costa, F. J. Lopez-Martinez, W. Hamouda, T. M. N. Nkouatchah and U. S. Dias, "Transmit Antenna Selection in Secure MIMO Systems Over \$lalphaImu\$ Fading Channels," IEEE Transactions on Communications, vol. 67, no. 9, pp. 64836498, Sept. 2019, doi: 10.1109/TCOMM.2019.2921966.

[4] M. Eskandari, A. M. Doost-Hoseini, J. Jung and I. Lee, "Antenna selection and power allocation for energy efficient MIMO systems," Journal of Communications and Networks, vol. 20, no. 6, pp. 546-553, Dec. 2018, doi: 10.1109/JCN.2018.000087.

[5] S. Asaad, A. Bereyhi, A. M. Rabiei, R. R. Müller and R. F. Schaefer, "Optimal Transmit Antenna Selection for Massive MIMO Wiretap Channels," IEEE Journal on Selected Areas in Communications, vol. 36, no. 4, pp. 817-828, April 2018, doi: 10.1109/JSAC.2018.2825159.

[6] D. Tran, H. Tran, D. Ha and G. Kaddoum, "Secure Transmit Antenna Selection Protocol for MIMO NOMA Networks Over Nakagami-m Channels," IEEE Systems Journal, vol. 14, no. 1, pp. 253-264, March 2020, doi: 10.1109/JSYST.2019.2900090. 
[7] D. He, C. Liu, T. Q. S. Quek and H. Wang, "Transmit Antenna Selection in MIMO Wiretap Channels: A Machine Learning Approach," in IEEE Wireless Communications Letters, vol. 7, no. 4, pp. 634-637, Aug. 2018, doi: 10.1109/LWC.2018.2805902.

[8] X. Yang and F. Zhao, "Multi-class import vector machine for transmit antenna selection in MIMO systems," in Electronics Letters, vol. 56, no. 2, pp. 62-65, 2301 2020, doi: 10.1049/el.2019.3233.

[9] Y. Gao, H. Vinck and T. Kaiser, "Massive MIMO Antenna Selection: Switching Architectures, Capacity Bounds, and Optimal Antenna Selection Algorithms," IEEE Transactions on Signal Processing, vol. 66, no. 5, pp. 1346-1360, March1, 12018.

[10] H. Tang and Z. Nie, "Massive MIMO antenna selection algorithms based on iterative swapping," Electronics Letters, vol. 54, no. 4, pp. 190-192, 2018.

[11] Bi-bo HU, Yuan-an LIU, Gang XIE, Jin-chun GAO, Ya-lin YANG, "Energy efficiency of massive MIMO wireless communication systems with antenna selection”, The Journal of China Universities of Posts and Telecommunications, vol. 21, no. 6, pp. 18, December 2014.

[12] Z. Liu, W. Du and D. Sun, "Energy and Spectral Efficiency Trade off for Massive MIMO Systems With Transmit Antenna Selection," IEEE Transactions on Vehicular Technology, vol. 66, no. 5, pp. 4453-4457, May 2017.

[13] M. Olyaee, M. Eslami and J. Haghighat, "An energy-efficient joint antenna and user selection algorithm for multi-user massive MIMO downlink," IET Communications, vol. 12, no. 3, pp. 255-260, 2202018.

[14] P. V. Amadori and C. Masouros, "Interference-Driven Antenna Selection for Massive Multiuser MIMO," IEEE Transactions on Vehicular Technology, vol. 65, no. 8, pp. 59445958, Aug. 2016. 
[15] H. Tang and Z. Nie, "RMV Antenna Selection Algorithm for Massive MIMO," IEEE Signal Processing Letters, vol. 25, no. 2, pp. 239-242, Feb. 2018.

[16] G. Xu, A. Liu, W. Jiang, H. Xiang and W. Luo, "Joint user scheduling and antenna selection in distributed massive MIMO systems with limited backhaul capacity," China Communications, vol. 11, no. 5, pp. 17-30, May 2014.

[17] Fangqi Zhu, Na Wu, Qilian Liang,"Channel estimation for massive MIMO with 2-D nested array deployment", Physical Communication, vol. 25, Part 2, pp. 432-437, December 2017.

[18] Mohammad Torabi, David Haccoun, "Effects of outdated channel state information on user scheduling in MRC MIMO systems with antenna selection”, Signal Processing, vol. 142, pp. 457-467, January 2018.

[19] Ramachandran Vijayarani, Lakshmanan Nithyanandan, "Selective Interference Rejection based Antenna Selection for MIMO over LTE Advanced Networks", Procedia Computer Science, vol. 93, pp. 624-631, 2016.

[20] Keerti Tiwari, Davinder S. Saini, Sunil V. Bhooshan, “Antenna selection for MIMO systems over Weibull-Gamma fading channel”, Perspectives in Science, vol. 8, pp. 475478, September 2016.

[21] Rizwan Khan, Azremi Abdullah Al-Hadi, Ping Jack Soh, Che Muhammad Nor Che Isa, Shahid Khan, "Dual polarized antennas with reduced user effects for LTE-U MIMO mobile terminals", AEU - International Journal of Electronics and Communications, vol. 111, November 2019, Article 152880.

[22] Aicha Mchbal, Naima Amar Touhami, Hanae Elftouh, Mahmoud Moubadir, Aziz Dkiouak, "Spatial and Polarization Diversity Performance Analysis of a Compact MIMO Antenna", Procedia Manufacturing, vol. 32, pp. 647-652, 2019. 
[23] Fatemeh Rezaei, Aliakbar Tadaion, "Multi-layer beamforming in uplink/downlink massive MIMO Systems with multi-antenna users”, Signal Processing, vol. 164, pp. 5866, November 2019.

[24] Masadeh, Raja \& Mahafzah, Basel \& Sharieh, Ahmad," Sea Lion Optimization Algorithm", International Journal of Advanced Computer Science and Applications, vol. no. 10 , pp. $388-395,2019$.

[25] Seyedali Mirjalili, Andrew Lewis, "The Whale Optimization Algorithm”, Advances in Engineering Software, vol. 95, pp. 51-67, May 2016.

[26] M. Marsaline Beno, Valarmathi I. R, Swamy S. M and B. R. Rajakumar, "Threshold prediction for segmenting tumour from brain MRI scans", International Journal of Imaging Systems and Technology, Vol. 24, No. 2, pp. 129-137, 2014, DOI: https://doi.org/10.1002/ima.22087.

[27] Inumula Veeraraghava Rao, V. Malleswara Rao," Optimal Transmit Antenna Selection Using Improved GSA in Massive MIMO Technology", Wireless Personal Communications, pp 1-1, 2019

[28] Rao, I.V., Rao, V.M. Massive MIMO perspective: improved sea lion for optimal antenna selection. Evol. Intel. (2020). https://doi.org/10.1007/s12065-020-00457-x.

[29] Nipanikar S I,Hima Deepthi V, "Enhanced Whale Optimization Algorithm and Wavelet Transform for Image Steganography", Multimedia Research, Vol.2,No.3, pp.2332,2019 .

[30] Inumula Veeraraghava Rao,Malleswara Rao V, "An Enhanced Whale Optimization Algorithm for Massive MIMO System", Journal of Networking and Communication Systems, Vol.2,No.4, pp.12-22,2019.

[31] Rupam Gupta Roy, "Rescheduling Based Congestion Management Method Using Hybrid Grey Wolf Optimization - Grasshopper Optimization Algorithm in Power 
System", Journal of Computational Mechanics, Power System and Control, Vol.2,No.1, pp.9-18, 2019. 